\title{
Lyapunov stability for discontinuous systems
}

\author{
Estabilidade de Lyapunov para sistemas descontínuos
}

\author{
Iguer Luis Domini dos Santos'
}

\begin{abstract}
The present work studies the stability analysis of equilibrium of ordinary differential equations with the discontinuous right side, also called discontinuous differential equations, using the notion of Carathéodory solution for differential equations. This way, it is studied the stability of equilibrium in the Lyapunov sense for discontinuous systems through nonsmooth Lyapunov functions. Then two existing Lyapunov theorems are obtained. The results established refer to systems determined by nonautonomous differential equations.
\end{abstract}

Keywords: Discontinuous differential equations; Carathéodory solutions; Lyapunov stability

\section{Resumo}

O presente trabalho estuda a análise de estabilidade do equilíbrio de equações diferenciais ordinárias com o lado direito descontínuo, também chamadas de equações diferenciais descontínuas, usando a noção de solução de Carathéodory para equações diferenciais. Desta forma, estuda-se a estabilidade do equilíbrio no sentido de Lyapunov para sistemas descontínuos através de funções de Lyapunov não suaves. Então, são obtidos dois teoremas de existência de Lyapunov. Os resultados estabelecidos referem-se a sistemas determinados por equações diferenciais não autônomas.

Palavras-chave: Equações diferenciais descontínuas; Soluções de Carathéodory; Estabilidade de Lyapunov 


\section{Introduction}

Discontinuous differential equations determine discontinuous systems. This report studies the stability in the sense of Lyapunov for discontinuous systems determined by discontinuous differential equations. So, it is studied the stability in the sense of Lyapunov of the equilibrium of discontinuous differential equations through nonsmooth Lyapunov functions. This study was also addressed by Shevitz e Paden (1994) and by Bacciotti e Ceragioli (2006), through different notions of generalized solutions to differential equations. Shevitz e Paden (1994) studies Lyapunov stability of equilibria by using the notion of Filippov solution, while Bacciotti e Ceragioli (2006) address Lyapunov stability of equilibria by using the notion of Carathéodory solution. The discontinuous systems addressed by Bacciotti e Ceragioli (2006) relate to autonomous differential equations. Motivated by Bacciotti e Ceragioli (2006), the present work studies Lyapunov stability of equilibria for discontinuous systems determined by nonautonomous differential equations, establishing stability properties that make an analogy with Theorem 3.1 given in Shevitz e Paden (1994).

Here are studied discontinuous systems determined by the nonautonomous differential equation

$$
\dot{x}(t)=f(t, x(t))
$$

where $x \in \mathbb{R}^{n}$ and $f: \mathbb{R} \times \mathbb{R}^{n} \rightarrow \mathbb{R}^{n}$. For the study of the stability properties of system (1), it is assumed throughout the work that for every $x_{0} \in \mathbb{R}^{n}$, there is at least one Carathéodory solution of differential equation (1) with the initial condition $x\left(t_{0}\right)=x_{0}$. It is also assumed that all Carathéodory solutions of (1) such that $x\left(t_{0}\right)=x_{0}$, are defined on $\left[t_{0},+\infty\right)$. The existence of Carathéodory solutions for (1) has been treated in Coddington e Levinson (1955) and Hale (1980).

Thus, it is studied stability properties of system (1) through nonsmooth Lyapunov functions. Then, sufficient conditions are obtained for the equilibrium to be uniformly stable, or uniformly asymptotically stable. That is, two existing Lyapunov theorems are obtained.

\section{Preliminaries}

In this section are considered basic concepts and results that will be used throughout the work.

\subsection{Lebesgue measurability}

The following are basic concepts of measure theory. A more complete approach to Lebesgue measure and integral can be found in Royden (1963).

Definition 2.1. A function $f: \mathbb{R}^{m} \rightarrow \mathbb{R}^{n}$ is said to be Lebesgue measurable if for all open set $V \subset \mathbb{R}^{n}$, the set

$$
f^{-1}(V)=\left\{x \in \mathbb{R}^{m}: f(x) \in V\right\}
$$

is Lebesgue measurable.

Let $I \subset \mathbb{R}$ be an interval. It is said that a statement $P$ holds almost everywhere (a.e.) on $I$, if the set $N$ given by

$$
N=\{t \in I: P \text { does not hold at } t\}
$$

has Lebesgue measure zero.

\subsection{Generalized solutions}

For completeness and ease of reading, Carathéodory and Filippov solutions for differential equation (1) are defined below. Solutions to the differential equation (1) will be understood as absolutely continuous functions. A approach to absolutely continuous functions can be found in Natanson, 1955 and Riesz e Sz.-Nagy (1955).

Definition 2.2. Let $I \subset \mathbb{R}$ be an interval. A function $x: I \rightarrow \mathbb{R}^{n}$ is a Carathéodory solution of (1) on $I$ if $x(t)$ is absolutely continuous and $\dot{x}(t)=f(t, x(t))$ for a.e. $t \in I$.

In the next definition, the function $f: \mathbb{R} \times \mathbb{R}^{n} \rightarrow \mathbb{R}^{n}$ is assumed to be Lebesgue measurable and essentially locally bounded.

Definition 2.3. A vector function $x(\cdot)$ is a Filippov solution of $(1)$ on $\left[t_{0}, t_{1}\right]$ if $x(\cdot)$ is absolutely continuous on $\left[t_{0}, t_{1}\right]$ and for a.e. $t \in\left[t_{0}, t_{1}\right]$

$$
\dot{x}(t) \in F_{F}(t, x(t))
$$


where

$$
F_{F}(t, x)=\bigcap_{\delta>0} \bigcap_{\mu(N)=0} \overline{c o}\{f(t, \mathcal{B}(x, \delta) \backslash N)\}
$$

( $\mu$ is the Lebesgue measure of $\mathbb{R}^{n}$, $\overline{\text { co }}$ denotes the closure of the convex hull, and $\mathcal{B}(x, r)$ is the open ball of radius $r$ centered at $x$ ).

Spraker e Biles (1996) address relations between the Carathéodory and Filippov solutions sets for the initial value problem

$$
\dot{x}(t)=f(x(t)), x(0)=x_{0}
$$

where $t \in[0, T]$ and $f: \mathbb{R}^{n} \rightarrow \mathbb{R}^{n}$. According to Spraker e Biles (1996), if $f: \mathbb{R} \rightarrow \mathbb{R}$ is given by

$$
f(x)= \begin{cases}0, & x=0 \\ 1, & x \neq 0\end{cases}
$$

and $x_{0}=0$, then $x(t) \equiv 0$ is a Carathéodory solution but not a Filippov solution to the initial value problem (2). On the other hand, if $f: \mathbb{R} \rightarrow \mathbb{R}$ is given by

$$
f(x)= \begin{cases}1, & x=0 \\ 0, & x \neq 0\end{cases}
$$

and $x_{0}=0$, then $x(t) \equiv 0$ is a Filippov solution but not a Carathéodory solution to the initial value problem (2).

\subsection{Generalized gradients}

Below is defined the Clarke's generalized gradient. It is proved here a chain rule (Theorem 2.1) similar to the chain rule given in [Shevitz e Paden (1994), Theorem 2.2].

Definition 2.4. For a locally Lipschitz function $V: \mathbb{R} \times \mathbb{R}^{n} \rightarrow \mathbb{R}$ define the generalized gradient of $V$ at $(t, x)$ by

$$
\partial V(t, x)=\overline{c o}\left\{\lim \nabla V\left(t_{i}, x_{i}\right) \mid\left(t_{i}, x_{i}\right) \rightarrow(t, x),\left(t_{i}, x_{i}\right) \notin \Omega_{V}\right\}
$$

where $\Omega_{V}$ is the set of measure zero where the gradient of $V$ is not defined.

Note that if $V: \mathbb{R} \times \mathbb{R}^{n} \rightarrow \mathbb{R}$ is given by $V(t, x)=V_{1}(x)$, where $V_{1}: \mathbb{R}^{n} \rightarrow \mathbb{R}$, then $\partial V(t, x)=\left(0, \partial V_{1}(x)\right)$.

Next, generalized directional derivative is defined.

Definition 2.5. The generalized directional derivative is defined by

$$
f^{\circ}(x ; v)=\limsup _{y \rightarrow x, t \downarrow 0} \frac{f(y+t v)-f(y)}{t} .
$$

The next result is due to Clarke (1983).

Lemma 2.1. Let $f$ be Lipschitz near $x$, then

$$
f^{\circ}(x ; v)=\max \{\langle\xi, v\rangle \mid \xi \in \partial f(x)\} .
$$

The chain rule (stated in Theorem 2.1) will be for the useful class of regular functions.

Definition 2.6. The function $f: \mathbb{R}^{n} \rightarrow \mathbb{R}$ is called regular if:

1) for all $v$, the usual one-sided directional derivative $f^{\prime}(x ; v)$ exists,

2) for all $v, f^{\prime}(x ; v)=f^{\circ}(x ; v)$.

Theorem 2.1. Let $x(\cdot)$ be a Carathéodory solution to (1) on an interval containing $t$. Suppose that the function $V: \mathbb{R} \times \mathbb{R}^{n} \rightarrow \mathbb{R}$ is Lipschitz and regular. Then $V(t, x(t))$ is absolutely continuous, $\frac{d}{d t} V(t, x(t))$ exists for a.e. $t$, and

$$
\frac{d}{d t} V(t, x(t))=\dot{\bar{V}}(t, x(t)) \quad \text { a.e. } t
$$

where

$$
\dot{\bar{V}}(t, x):=\bigcap_{\xi \in \partial V(t, x)}\langle\xi,(1, f(t, x))\rangle .
$$


Proof. Since $V$ is a Lipschitz function and $(t, x(t))$ is an absolutely continuous function, then $V(t, x(t))$ is absolutely continuous. Hence, $\frac{d}{d t} V(t, x(t))$ exists for a.e. $t$. In what follows, $o(h)$ is a real function such that $\lim _{h \rightarrow 0} \frac{o(h)}{|h|}=0$. If $t$ is a point where $x(t)$ and $V(t, x(t))$ are both differentiable (this is holds a.e. $t$ ), then

$$
\begin{aligned}
\frac{d}{d t} V(t, x(t)) & =\lim _{h \downarrow 0} \frac{V(t+h, x(t+h))-V(t, x(t))}{h} \\
& =\lim _{h \downarrow 0} \frac{V(t+h, x(t)+\dot{x}(t) h)+o(h)-V(t, x(t))}{h} \\
& =V^{\prime}((t, x(t)) ;(1, \dot{x}(t))) \\
& =V^{\circ}((t, x(t)) ;(1, \dot{x}(t))) \\
& =\max \{\langle\xi,(1, \dot{x}(t))\rangle \mid \xi \in \partial V(t, x(t))\}
\end{aligned}
$$

and

$$
\begin{aligned}
\frac{d}{d t} V(t, x(t)) & =\lim _{h \downarrow 0} \frac{V(t-h, x(t-h))-V(t, x(t))}{-h} \\
& =\lim _{h \downarrow 0} \frac{V(t-h, x(t)-\dot{x}(t) h)+o(h)-V(t, x(t))}{-h} \\
& =-V^{\prime}((t, x(t)) ;(-1,-\dot{x}(t))) \\
& =-V^{\circ}((t, x(t)) ;(-1,-\dot{x}(t))) \\
& =-\max \{\langle\xi,(-1,-\dot{x}(t))\rangle \mid \xi \in \partial V(t, x(t))\} \\
& =\min \{\langle\xi,(1, \dot{x}(t))\rangle \mid \xi \in \partial V(t, x(t))\} .
\end{aligned}
$$

Therefore,

$$
\frac{d}{d t} V(t, x(t))=\bigcap_{\xi \in \partial V(t, x(t))}\langle\xi,(1, \dot{x}(t))\rangle \text { a.e.t. }
$$

Since $x(\cdot)$ is a Carathéodory solution to (1), it follows that

$$
\frac{d}{d t} V(t, x(t))=\bigcap_{\xi \in \partial V(t, x(t))}\langle\xi,(1, f(t, x(t)))\rangle \text { a.e. } t,
$$

that is,

$$
\frac{d}{d t} V(t, x(t))=\dot{\bar{V}}(t, x(t)) \quad \text { a.e. } t \text {. }
$$

For systems determined by autonomous differential equations, the following chain rule applies.

Proposition 2.1. Let $x(\cdot)$ be a Carathéodory solution to $\dot{x}(t)=f(x(t))$ on an interval containing $t$. Suppose that the function $V: \mathbb{R}^{n} \rightarrow \mathbb{R}$ is Lipschitz and regular. Then $V(x(t))$ is absolutely continuous, $\frac{d}{d t} V(x(t))$ exists for a.e. $t$, and

$$
\frac{d}{d t} V(x(t))=\dot{\bar{V}}(x(t)) \quad \text { a.e. } t
$$

where

$$
\dot{\bar{V}}(x):=\bigcap_{\xi \in \partial V(x)}\langle\xi, f(x)\rangle .
$$

\subsection{Nonpathological functions}

Nonpathological functions will be considered here because of Examples 3.1 and 3.2. The considerations made here about nonpathological functions are based on Bacciotti e Ceragioli (2006). 
Definition 2.7. It is said that a function $V: \mathbb{R}^{n} \rightarrow \mathbb{R}$ is nonpathological if it is locally Lipschitz continuous and for every absolutely continuous function $\varphi: I \subset \mathbb{R} \rightarrow \mathbb{R}^{n}$ and for a.e. $t \in I$, the set $\partial V(\varphi(t))$ is a subset of an affine subspace orthogonal to $\dot{\varphi}(t)$.

Bacciotti e Ceragioli (2006) use nonpathological functions like Lyapunov functions to obtain stability properties (see for instance [Bacciotti e Ceragioli (2006), Proposition 2]).

Proposition 2.2 (Bacciotti e Ceragioli (2006)). If $V: \mathbb{R}^{n} \rightarrow \mathbb{R}$ is nonpathological, and $\varphi: \mathbb{R} \rightarrow \mathbb{R}^{n}$ is absolutely continuous, then the set $\{\langle p, \dot{\varphi}(t)\rangle: p \in \partial V(\varphi(t))\}$ is reduced to the singleton $\left\{\frac{d}{d t} V(\varphi(t))\right\}$ for a.e. $t$.

Corollary 2.1 (Bacciotti e Ceragioli (2006)). Let the function $V: \mathbb{R}^{n} \rightarrow \mathbb{R}$ be nonpathological, and let $\varphi$ be any solution of system $\dot{x}=f(x)$. Then

$$
\varphi(t) \in\left\{x \in \mathbb{R}^{n}:\left\langle p_{1}, f(x)\right\rangle=\left\langle p_{2}, f(x)\right\rangle, \forall p_{1}, p_{2} \in \partial V(x)\right\}
$$

and $\frac{d}{d t} V(\varphi(t))=\langle p, f(\varphi(t))\rangle$ for any $p \in \partial V(\varphi(t))$, and for a.e. $t$.

\section{Stability properties}

In this section, it is proved stability properties to system (1). The contributions of the paper to the Lyapunov's stability theory to discontinuous systems are stated in Theorem 3.1.

Let the set of Carathéodory solutions of (1) with initial condition $x\left(t_{0}\right)=x_{0}$ be denoted by $S\left(x_{0}, t_{0}\right)$. Suppose that for any initial conditions $\left(x_{0}, t_{0}\right)$ the set $S\left(x_{0}, t_{0}\right)$ is nonempty and all solutions are defined on the interval $\left[t_{0}, \infty\right)$. Also suppose that the origin $x=0$ is an equilibrium point of (1) at $t=0$, that is, $f(t, 0)=0$ for all $t \geq 0$.

Below are defined concepts of stability in the Lyapunov sense.

Definition 3.1. The equilibrium point $x=0$ of (1) is stable, if for any $\varepsilon>0$ and any $t_{0} \geq 0$, there is a $\delta=\delta\left(\varepsilon, t_{0}\right)>0$ such that $\left|x_{0}\right|<\delta$ implies $|x(t)|<\varepsilon$ for $t \in\left[t_{0}, \infty\right)$ and $x(t) \in S\left(x_{0}, t_{0}\right)$.

If in the previous definition $\delta$ is independent of $t_{0}$, that is, if $\delta=\delta(\varepsilon)$, then the equilibrium point $x=0$ of (1) is said to be uniformly stable.

Definition 3.2. The equilibrium point $x=0$ of (1) is uniformly asymptotically stable if it is uniformly stable, and there is a $\delta_{0}>0$ such that for every $\varepsilon>0$ and for any $t_{0} \geq 0$, there exists a $T(\varepsilon)>0$, independent of $t_{0}$, such that $\left|x_{0}\right|<\delta_{0}$ implies $|x(t)|<\varepsilon$ if $t \geq t_{0}+T(\varepsilon)$ and $x(t) \in S\left(x_{0}, t_{0}\right)$.

Theorem 3.1 given below states two existing Lyapunov theorems (uniform stability and uniform asymptotic stability) in terms of $\dot{\bar{V}}$. The concept of class $\mathcal{K}$ function considered below can be found in Khalil (2002).

Theorem 3.1. Let $V: \mathbb{R} \times \mathbb{R}^{n} \rightarrow \mathbb{R}$ be a locally Lipschitz continuous and regular function. Suppose there exist functions $\psi_{1}$ and $\psi_{2} \in \mathcal{K}$ satisfying

$$
\psi_{1}(|x|) \leq V(t, x) \leq \psi_{2}(|x|)
$$

for all $t \geq 0$ and for all $x$ with $|x| \leq r$. Thence:

1) If $\dot{\bar{V}}(t, x(t)) \leq 0$ for all $t \geq 0$ and for all Carathéodory solution $x(t)$ of (1) with $|x(t)| \leq r$, then the equilibrium point $x=0$ is uniformly stable.

2) If in addition, there exists a function $\psi_{3} \in \mathcal{K}$ obeying

$$
\dot{\bar{V}}(t, x(t)) \leq-\psi_{3}(|x(t)|)
$$

for all $t \geq 0$ and for all Carathéodory solution $x(t)$ of $(1)$ with $|x(t)| \leq r$, then the equilibrium point $x=0$ is uniformly asymptotically stable.

The proofs of Theorem 3.1 are similar to their smooth counterparts. The smooth version of item 1 for Theorem 3.1 can be founded in [Vidyasagar (1993), Theorem (5.3.14)] and [Miller e Michel (1982), Theorem 9.2]. On the other hand, the smooth version of item 2 for Theorem 3.1 can be founded in [Vidyasagar (1993), Theorem (5.3.25)] and [Miller e Michel (1982), Theorem 9.6].

Theorem 3.1 remains valid if the expression "for all $t \geq 0$ " in items 1 and 2 is replaced by "for a.e. $t \geq 0$ ", in this case, by Theorem 2.1, $\dot{\bar{V}}(t, x(t))$ can be replaced by $\frac{d}{d t} V(t, x(t))$.

In the following, it is also stated a result analogous to Theorem 3.1 for systems determined by autonomous differential equations. 
Theorem 3.2. Let $V: \mathbb{R}^{n} \rightarrow \mathbb{R}$ be a locally Lipschitz continuous and regular function. Suppose there exist functions $\psi_{1}$ and $\psi_{2} \in \mathcal{K}$ such that

$$
\psi_{1}(|x|) \leq V(x) \leq \psi_{2}(|x|)
$$

for all $x$ with $|x| \leq r$. Thence:

1) If $\dot{\bar{V}}(x(t)) \leq 0$ for all $t \geq 0$ and for all Carathéodory solution $x(t)$ to $\dot{x}(t)=f(x(t))$ with $|x(t)| \leq r$, then the equilibrium point $x=0$ is uniformly stable.

2) If in addition, there exists a function $\psi_{3} \in \mathcal{K}$ satisfying

$$
\dot{\bar{V}}(x(t)) \leq-\psi_{3}(|x(t)|)
$$

for all $t \geq 0$ and for all Carathéodory solution $x(t)$ to $\dot{x}(t)=f(x(t))$ with $|x(t)| \leq r$, then the equilibrium point $x=0$ is uniformly asymptotically stable.

The following are two examples for using the results obtained in this section.

Example 3.1. Consider the Artstein's circles example

$$
\left\{\begin{array}{l}
\dot{x}=\left(x^{2}-y^{2}\right) u \\
\dot{y}=2 x y u
\end{array}\right.
$$

where $u \in \mathbb{R}$. Similarly to [Bacciotti e Ceragioli (2006), Example 1], let

$$
u(x, y)= \begin{cases}1, & x<0 \\ -1, & x \geq 0\end{cases}
$$

Then the equilibrium point $(x, y)=(0,0)$ of (3) is uniformly stable.

Proof. Let $V: \mathbb{R}^{n} \rightarrow \mathbb{R}$ be the nonpathological function $V(x, y)=\sqrt{4 x^{2}+3 y^{2}}-|x|$. Although the function $V$ is not regular (in $x=0$ ), it is still possible to use Theorem 3.2 because of Proposition 2.2 and Corollary 2.1. Take an arbitrary Carathéodory solution $(x(t), y(t))$ of (3), and then consider

$$
\dot{\bar{V}}(x(t), y(t))=\bigcap_{\xi \in \partial V(x(t), y(t))}\langle\xi, f(x(t), y(t))\rangle
$$

where $f(x, y)=\left(\left(x^{2}-y^{2}\right) u, 2 x y u\right)$. If $t \geq 0$ is such that $x(t) \neq 0$, it follows that $\partial V(x(t), y(t))=\nabla V(x(t), y(t))$. Hence $\dot{\bar{V}}(x(t), y(t)) \leq 0$, since

$$
\langle\nabla V(x, y), f(x, y)\rangle=\frac{-4|x|^{3}-2|x| y^{2}+\left(x^{2}-y^{2}\right) \sqrt{4 x^{2}+3 y^{2}}}{\sqrt{4 x^{2}+3 y^{2}}} \leq 0
$$

whenever $x \neq 0$. On the other hand, suppose that $t \geq 0$ is such that $x(t)=0$. If $y(t)>0$,

$$
\left(0, \frac{3}{\sqrt{3}}\right) \in \partial V(0, y(t))=\left\{\left(s, \frac{3}{\sqrt{3}}\right): s \in[-1,1]\right\}
$$

and if $y(t)<0$,

$$
\left(0,-\frac{3}{\sqrt{3}}\right) \in \partial V(0, y(t))=\left\{\left(s,-\frac{3}{\sqrt{3}}\right): s \in[-1,1]\right\} .
$$

Now, if $y(t)=0$ then

$$
(0,0) \in \partial V(0,0)=\left\{(s, v): s \in[-1,1], v \in\left[-\frac{3}{\sqrt{3}}, \frac{3}{\sqrt{3}}\right]\right\}
$$

In any case, if $x(t)=0$ it follows that $f(x(t), y(t))=\left(-y(t)^{2} u, 0\right)$ and so

$$
\dot{\bar{V}}(x(t), y(t))=0 \text {. }
$$

Therefore $\dot{\bar{V}}(x(t), y(t)) \leq 0$ for all $t \geq 0$ to an arbitrary Carathéodory solution $(x(t), y(t))$ of (3). It follows from Theorem 3.2 that the equilibrium point $(x, y)=(0,0)$ of $(3)$ is uniformly stable. 
The system of differential equations given in the example below was treated in Grzanek et al. (2008).

Example 3.2. Consider the two dimensional system

$$
\left\{\begin{array}{l}
\dot{x}=-x f_{2}(t, x, y)+|y| \\
\dot{y}=-y f_{1}(t, x, y)+|x|
\end{array}\right.
$$

where $f_{1}, f_{2}:[0, \infty) \times \mathbb{R}^{2} \rightarrow \mathbb{R}, f_{2}(t, x, y) \geq \operatorname{sgn}(y)$ and $f_{1}(t, x, y) \geq \operatorname{sgn}(x)$. Then the equilibrium point $(x, y)=(0,0)$ of $(4)$ is uniformly stable.

Proof. Let $V: \mathbb{R} \times \mathbb{R}^{n} \rightarrow \mathbb{R}$ be defined as $V(t, x, y)=V_{1}(x, y)$, where $V_{1}(x, y)=|x|+|y|$. The function $V$ is not regular (whenever $x=0$ or $y=0$ ), but $V_{1}$ is nonpathological. Hence, it is still possible to use Theorem 3.1 because of Proposition 2.2. Let $(x(t), y(t))$ be an arbitrary Carathéodory solution of (4) and let

$$
\begin{aligned}
\dot{\bar{V}}(t, x(t), y(t)) & =\bigcap_{\xi \in \partial V(t, x(t), y(t))}\langle\xi,(1, f(t, x(t), y(t)))\rangle \\
& =\bigcap_{\xi \in\left(0, \partial V_{1}(x(t), y(t))\right)}\langle\xi,(1, f(t, x(t), y(t)))\rangle \\
& =\bigcap_{\left.\eta \in \partial V_{1}(x(t), y(t))\right)}\langle\eta, f(t, x(t), y(t))\rangle
\end{aligned}
$$

where $f(t, x, y)=\left(-x f_{2}(t, x, y)+|y|,-y f_{1}(t, x, y)+|x|\right)$. For brevity, denote $x(t)$ by $x$ and $y(t)$ by $y$. If $t \geq 0$ is such that $x(t) \neq 0$ and $y(t) \neq 0$, it follows that $\partial V(t, x, y)=\left(0, \nabla V_{1}(x, y)\right)=(0, \operatorname{sgn}(x), \operatorname{sgn}(y))$. Thence $\dot{\bar{V}}(t, x, y) \leq 0$, since

$$
\begin{aligned}
\langle(0, \operatorname{sgn}(x), \operatorname{sgn}(y)),(1, f(t, x, y))\rangle & =\operatorname{sgn}(x)\left(-x f_{2}(t, x, y)+|y|\right)+\operatorname{sgn}(y)\left(-y f_{1}(t, x, y)+|x|\right) \\
& =-|x| f_{2}(t, x, y)+\operatorname{sgn}(x)|y|-|y| f_{1}(t, x, y)+\operatorname{sgn}(y)|x| \\
& =-|x|\left(f_{2}(t, x, y)-\operatorname{sgn}(y)\right)-|y|\left(f_{1}(t, x, y)-\operatorname{sgn}(x)\right) \leq 0 .
\end{aligned}
$$

Now, suppose that $t \geq 0$ is such that $y(t)=0$. If $x(t)>0$,

$$
\left.(1,0) \in \partial V_{1}(x(t), 0)\right)=\{(1, s): s \in[-1,1]\}
$$

and thus

$$
\begin{aligned}
\dot{\bar{V}}(t, x(t), y(t)) & =\left\langle(1,0),\left(-x f_{2}(t, x, 0),|x|\right)\right\rangle \\
& =-x f_{2}(t, x, 0) \leq 0
\end{aligned}
$$

because $f_{2}(t, x, 0) \geq \operatorname{sgn}(0)=0$. If $x(t)<0$,

$$
\left.(-1,0) \in \partial V_{1}(x(t), 0)\right)=\{(-1, s): s \in[-1,1]\}
$$

and thus

$$
\begin{aligned}
\dot{\bar{V}}(t, x(t), y(t)) & =\left\langle(-1,0),\left(-x f_{2}(t, x, 0),|x|\right)\right\rangle \\
& =x f_{2}(t, x, 0) \leq 0 .
\end{aligned}
$$

On the other hand, suppose that $t \geq 0$ is such that $x(t)=0$. If $y(t)>0$,

$$
\left.(0,1) \in \partial V_{1}(0, y(t))\right)=\{(s, 1): s \in[-1,1]\}
$$

and then

$$
\begin{aligned}
\dot{\bar{V}}(t, x(t), y(t)) & =\left\langle(0,1),\left(|y|,-y f_{1}(t, x, y)\right)\right\rangle \\
& =-y f_{1}(t, x, y) \leq 0
\end{aligned}
$$

since $f_{1}(t, 0, y) \geq \operatorname{sgn}(0)=0$. If $y(t)<0$,

$$
\left.(0,-1) \in \partial V_{1}(0, y(t))\right)=\{(s,-1): s \in[-1,1]\}
$$


and then

$$
\begin{aligned}
\dot{\bar{V}}(t, x(t), y(t)) & =\left\langle(0,-1),\left(|y|,-y f_{1}(t, x, y)\right)\right\rangle \\
& =y f_{1}(t, x, y) \leq 0 .
\end{aligned}
$$

Finally, if $t \geq 0$ is such that $(x(t), y(t))=(0,0)$,

$$
\left.\left.(0,0) \in \partial V_{1}(0,0)\right)\right)=\{(s, v): s \in[-1,1], v \in[-1,1]\}
$$

and then $\dot{\bar{V}}(t, x(t), y(t))=0$. Therefore $\dot{\bar{V}}(t, x(t), y(t)) \leq 0$ for all $t \geq 0$ to an arbitrary Carathéodory solution $(x(t), y(t))$ of $(4)$. From Theorem 3.1 the equilibrium point $(x, y)=(0,0)$ of (4) is uniformly stable.

\section{Conclusions}

The paper contributes to the Lyapunov's stability theory to discontinuous systems. More specifically, the paper studies stability properties for discontinuous systems determined by discontinuous differential equations. The stability properties studied here relate to uniform stability and uniform asymptotic stability (Theorem 3.1 and Theorem 3.2). Theorem 3.1 establishes stability properties for systems determined by autonomous differential equations, while Theorem 3.2 establishes stability properties for systems determined by nonautonomous differential equations.

\section{References}

Bacciotti, A., Ceragioli, F. (2006). Nonpathological Lyapunov functions and discontinuous Carathéodory systems. Automatica, 42(3), 453-458.

Clarke, F. H. (1983). Optimization and nonsmooth analysis. John Wiley \& Sons, Inc., New York.

Coddington, E. A., Levinson, N. (1955). Theory of ordinary differential equations. McGraw-Hill Book Company, Inc., New York-Toronto-London.

Grzanek, M., Michalak, A., Rogowski, A. (2008). A nonsmooth Lyapunov function and stability for ODE's of Carathéodory type. Nonlinear Anal, 69(1), 337-342.

Hale, J. K. (1980). Ordinary differential equations, $2^{\circ}$ edn. Robert E. Krieger Publishing Co., Inc., Huntington, N.Y..

Khalil, H. K. (2002). Nonlinear systems. 3rd ed., $3^{\circ}$ edn. Upper Saddle River, NJ: Prentice Hall.

Miller, R. K., Michel, A. N. (1982). Ordinary differential equations. Academic Press, Inc. [Harcourt Brace Jovanovich, Publishers], New York-London.

Natanson, I. P. (1955). Theory of functions of a real variable. Frederick Ungar Publishing Co., New York.

Riesz, F., Sz.-Nagy, B. (1955). Functional analysis. Frederick Ungar Publishing Co., New York.

Royden, H. L. (1963). Real analysis. The Macmillan Co., New York; Collier-Macmillan Ltd., London.

Shevitz, D., Paden, B. (1994). Lyapunov stability theory of nonsmooth systems. IEEE Trans Automat Control, 39(9), 1910-1914.

Spraker, J. S., Biles, D. C. (1996). A comparison of the Carathéodory and Filippov solution sets. J Math Anal Appl, 198(2), 571-580.

Vidyasagar, M. (1993). Nonlinear systems analysis. 2nd ed., $2^{\circ}$ edn. London: Prentice Hall. 